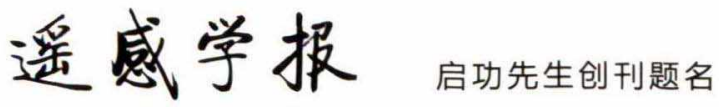

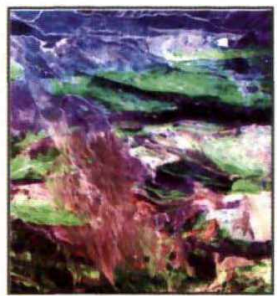

\author{
封面说明 \\ About the Cover \\ GF-5 AHSI新疆东天山高光谱数据地质精细特征分类图 \\ GF-5 AHSI geological characteristics map in East Tianshan, Xinjiang
}

高分五号卫星 (GF-5) 是世界首颗实现对大气和陆地综合观测的全谱段高光谱卫星，其上搭载的可见短波红外高光谱相机 (AHSI) 是国际上首台具有自主 知识产权的宽谱宽幅星载高光谱成像载荷。封面图片为 GF-5 AHSI 于 2019-03-20 获取的新疆哈密东天山各地层及岩体等高光谱数据地质特征分类图。通 过高光谱数据提取的 32 个主类，划分为不同颜色，有效区分各地层砂岩，变质岩，中基性到酸性岩体，蚀变带等地质特征信息，可识别出多个已知的矿区 (点) 信息，以及一些未知矿化信息异常区，这标志着利用高光谱数据在全球大范围能源矿产资源定量化探测成为可能。GF-5 AHSI 已广泛应用于地质岩性 矿物精细填图及油气资源勘查、生态环境与水质监测、土壤肥力及重金属含量反演、作物长势及产量预测、植被精细分类及生产力评估、海洋与海岸带监 测等领域。

GF-5 is the world's first full-spectrum hyperspectral satellite to achieve comprehensive observations of the atmosphere and land. The visible short-wave infrared hyperspectral camera (AHSI) mounted on GF-5 is the world's first wide-spectrum wide-swath spaceborne hyperspectral imaging payload with independent intellectual property. The cover image shows the geological feature classification map of hyperspectral data of layers and rock masses in East Tianshan in Hami, Xinjiang, which was acquired by AHSI on March 20, 2019. The 32 main categories extracted from hyperspectral data are divided into different colors, which effectively distinguish geological characteristics such as sandstone, metamorphic rock, medium basic to acidic rock masses, and alteration zone in various layers. It can identify multiple known information of mineral area or mineral spot, as well as some anomalous areas of unknown mineralization information.GF-5 AHSI is widely used in fine mapping of lithologic geological minerals and mineral resources, exploration of oil and gas resources, ecological environment and water quality detection, soil fertility retrieval of heavy metal content, crop growth and yield prediction, fine vegetation classification and productivity assessment, marine and coastal zone monitoring, etc.

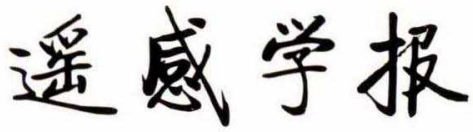

YAOGAN XUEBAO（月刊 1997年创刊）

第24卷 第4期 2020年4月25日

\section{JOURNAL OF REMOTE SENSING}

(Monthly, Published since 1997)

Vol.24 No.4 April 25, 2020

\begin{tabular}{|c|c|c|c|c|}
\hline 主＼cjkstart管 & 中国科学院 & Superintended & by & Chinese Academy of Sciences \\
\hline 办 & $\begin{array}{l}\text { 中国科学院遥感与数字地球研究所 } \\
\text { 中国地理学会环境遥感分会 }\end{array}$ & Sponsored & by & $\begin{array}{l}\text { Institute of Remote Sensing and Digital Earth,CAS } \\
\text { The Associate on Environment Remote Sensing } \\
\text { of China }\end{array}$ \\
\hline 主编 & 顾行发 & Editor in Chief & & GU Xingfa \\
\hline 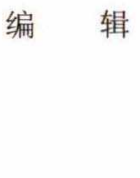 & $\begin{array}{l}\text { 《遥感学报》编委会 } \\
\text { 北京市海淀区北四环西路19号 } \\
\text { 邮编 : } 100190 \text { 电话 : 86-10-58887052 } \\
\text { http://www.jors.cn } \\
\text { E-mail: jrs@radi.ac.cn }\end{array}$ & Edited & by & $\begin{array}{l}\text { Editorial Board of Journal of Remote Sensing } \\
\text { Add: P.O.Box 2702, Beijing 100190, China } \\
\text { Tel: } 86-10-58887052 \\
\text { http://www.jors.cn } \\
\text { E-mail: jrs@radi.ac.cn }\end{array}$ \\
\hline $\begin{array}{l}\text { 出版 } \\
\text { 印刷装订 }\end{array}$ & $\begin{array}{l}\text { 料学出馧社 } \\
\text { 北京科信印刷有限公司 }\end{array}$ & $\begin{array}{l}\text { Published } \\
\text { Printed }\end{array}$ & $\begin{array}{l}\text { by } \\
\text { by }\end{array}$ & $\begin{array}{l}\text { Science Press } \\
\text { Beijing Kexin Printing Co. Ltd. }\end{array}$ \\
\hline 总 发 行 & $\begin{array}{l}\text { 科学出弼社 } \\
\text { 北京东黄城根北街16号 国内邮发代号: 82-324 } \\
\text { 邮政编码 : } 100717 \\
\text { 电话: } 86-10-64017032 \\
\text { 淘宝店铺名称: 中科期刊 }\end{array}$ & Distributed & by & $\begin{array}{l}\text { Science Press } \\
\text { Add: } 16 \text { Donghuangchenggen North Street, } \\
\quad \text { Beijing } 100717 \text {, China } \\
\text { Tel: } 86-10-64017032 \\
\text { Taobao:Zhongke Journal }\end{array}$ \\
\hline 国外发行 & $\begin{array}{l}\text { 中国国际图书贸易总公司 } \\
\text { 北京 } 399 \text { 信箱 邮政编码 : } 100044 \text { 国外发行代号: BM } 1002\end{array}$ & Overseas distributed & by & $\begin{array}{l}\text { China International Book Trading Corporation } \\
\text { Add: P.O.Box } 399 \text {, Beijing 100044, China }\end{array}$ \\
\hline
\end{tabular}

中国标准连续出版物号： ISSN 1007-4619

CODEN YXAUAB CN 11-3841/TP

eISSN 2095-9494

定价：70.00元

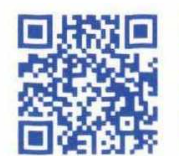

官网

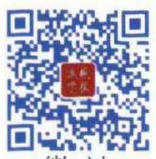

微站

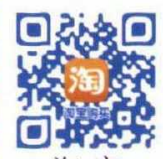

淘宝

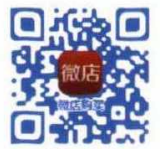

微 店
I S S N $1007-4619$

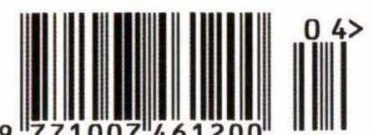

